\title{
A New HRRP Reconstruction Method based on Joint Sparse Model
}

\author{
Zhou Ming a , Lv Mingjiu \\ Air Force Early Warning Academy,Wuhan,China,430010 \\ aemail:yasha1234@163.com
}

Key words:High resolution range profile, Joint Sparse Model, Random Sparse stepping frequency Signal, compressed sensing.

\begin{abstract}
At present, most of the high resolution range profile reconstruction methods are based on a single echo signal, without considering the relationship between all the echo signals. In this paper, we consider the sparse feature of echo signal, and propose a method of high resolution range profile reconstruction based on joint sparse model. The method can not only improve the accuracy of the reconstruction, but also reduce the number of the reconstructed volume, and have strong anti-noise performance. Finally, the advantages of the proposed method in this paper are demonstrated by simulation and analysis.
\end{abstract}

\section{Introduction}

Traditional step frequency signal by transmitting frequency stepping pulse signal to achieve high range resolution, but must be emission many sub-pulses whose carrier frequency are continuous variation, the imaging time is longer, when to get the two-dimensional image, the time required will be longer. Sparse stepping frequency (SSF) signal by randomly emitting portion sub-pulses of traditional stepping frequency signal to achieve high range resolution capability, it can greatly reducing the imaging time. In order to increase the signal anti-jamming performance, the variation of the carrier frequency is not the same in different groups. However, for the traditional step frequency signal, the HRRP can be obtained by IFFT processing, but it is not suitable for this RSSF signal. How to deal with this kind of signal has been widely concerned by scholars.

The introduction of compressed sensing (CS) theory provides an effective way to deal with this kind of signal. The compressed sensing reconstruction method for sparse step frequency signal is studied in Literature [1,2]. Literature [3] studied the Bayesian reconstruction algorithm for sparse step frequency ISAR signal. Literature [4] studied the compression step stochastic FM frequency signal sensing imaging methods. Literature [5] studied the sparse step frequency signal imaging method based on multiple measurement vector model. However, most of the compressed sensing methods at present are based on each pulse to reconstruct the HRRP, without considering the relationship between the signal groups. In fact, because of the sparse nature of the target in the observation area, all the radar echoes have the same sparse structure as the radar observation target. Based on sparse model, a new HRRP reconstruction method is studied in this paper. when reconstructed the HRRP, using the joint sparse characteristic of signals, which improves the reconstruction accuracy and reduces the number of the measured values, and enhances the anti-noise performance. Theoretical analysis and simulation results verify the effectiveness of the proposed algorithm.

\section{The RSSF echo model based on JSM}

Traditional step frequency signal is mainly consists of a series of carrier frequency continuous changes in the sub pulses, adjacent sub carrier frequency pulse with fixed frequency step $(\Delta f)$.Each pulse train contains n sub-pulses. Then, $N_{a}$ groups of the fundamental frequency echo signal can be expressed as ${ }^{[6]}$ :

$$
S_{R}(t)=\sum_{i=1}^{I} \sigma_{i} \sum_{n_{a}=0}^{N_{a}-1} \sum_{n=0}^{N-1} r e c t\left[\frac{t-n T_{r}-n_{a} N T_{r}-2 R(t) / c}{T}\right] \exp \left[-j 4 \pi f_{n} R_{i}\left(t, n_{a}\right) / c\right]
$$


$\operatorname{rect}(u)=\left\{\begin{array}{ll}1, & |u| \leq 1 / 2 \\ 0, & |u|>1 / 2\end{array}\right.$ indicates rectangular window, $\sigma_{i}, i=1, \ldots . . . I$ represents the scattering point intensity of target, $t$ is the whole time; $N$ is the number of sub-pulse; $T_{r}$ is pulse repetition interval; $T$ is pulse width; $f_{0}$ is carrier frequency; $\Delta f$ is step frequency; $R_{i}\left(t, n_{a}\right)$ represents the distance between radar and target scattering point . The variation of the signal carrier frequency can be expressed as $f_{n}=f_{0}+n \Delta f$.

For the RSSF signal, which the carrier frequency variation can be seen as a carrier in $N$ were randomly selected for $M$ emission. For $N_{a}$ groups of random sparse stepping frequency signal, which the carrier frequency variation can be expressed as $f_{m, n_{a}}=f_{0}+\left(m, n_{a}\right) \Delta f, \quad\left(m, n_{a}\right)$ is random positive integer representing the sub-pulses' stepping ratio of $n_{a}$ group. Then, the fundamental frequency echo signal can be expressed as:

$$
S_{R}(t)=\sum_{i=1}^{I} \sigma_{i} \sum_{n_{a}=0}^{N_{a}-1} \sum_{m=0}^{M-1} \operatorname{rect}\left[\frac{t-m T_{r}-n_{a} N T_{r}-2 R(t) / c}{T}\right] \exp \left[-j 4 \pi f_{m, n_{a}} R_{i}\left(t, n_{a}\right) / c\right]
$$

Assumed the target motion compensation has been completed. Then $R_{i}\left(t, n_{a}\right)$ can be expressed as:

$$
R_{i}\left(t, n_{a}\right)=R_{0}+x_{i} \cos \left[\theta\left(n_{a}\right)\right]-y_{i} \sin \left[\theta\left(n_{a}\right)\right]
$$

In the above formula, $\theta\left(n_{a}\right) \approx n_{a} \Delta \theta, \Delta \theta$ is rotating step, $R_{0}$ is the distance between radar and target reference point.

In general, the radar imaging time is very short, so $\cos \left[\theta\left(n_{a}\right)\right] \approx 1, \sin \left[\theta\left(n_{a}\right)\right] \approx n_{a} \Delta \theta$. Then we can obtain the final sampling signal like this:

$$
U_{m, n_{a}}=\sum_{i=1}^{I} \sigma_{i} \exp \left[-j 4 \pi f_{m, n_{a}} \frac{x_{i}-y_{i} n_{a} \Delta \theta}{c}\right]
$$

\section{HRRP imaging method based on JSM}

The high resolution range imaging method based on JSM is studied in this section.

For the echo, because the observation of the target in the observation area can be regarded as sparse, so it is also a sparse signal, just as the visual angle, noise and other factors, makes the non-zero position amplitude difference. The above-mentioned properties can be expressed as:

$$
\begin{aligned}
& \Gamma_{1}=\Gamma_{2}=\ldots=\Gamma_{N_{a}} \\
& \left\|\Gamma_{n_{a}}\right\|_{0}=K, \forall n_{a} \in\left\{1,2, \ldots, N_{a}\right\}
\end{aligned}
$$

In solving the model, the characteristics of echo signal joint sparse can be used as prior information, improve the reconstruction accuracy.

The RSSF's sampling echo signal can be used as the observation signal, and the HRRP of the target can be reconstructed by using the theory of compressed sensing. The process is as follows:

$$
U_{m, n_{a}}=\Phi_{n_{a}} U\left(m ; n_{a}\right)=\Theta_{n_{a}} \operatorname{HRRP}\left(n_{a}\right)
$$

Where $U\left(m ; n_{a}\right)$ is the traditional stepping frequency echo data; $\operatorname{HRRP}\left(n, n_{a}\right)$ represents the high resolution range; $\Theta_{n_{a}}=\Phi_{n_{a}} \Psi, \Phi_{n_{a}}$ is the measurement matrix; $\Psi$ is the sparse basis of the distance.

In accordance with the rules of sub pulse design $M \times N$ dimensional random measurement matrix $\Phi_{n_{a}}=\left\{\phi_{m, n}\right\}$ as follows:

$$
\phi_{m, n}=\left\{\begin{array}{cc}
1, \quad m=\left(m, n_{a}\right) \\
0, \quad \text { others }
\end{array}\right.
$$

The traditional step frequency signal through the IDFT transform to get HRRP of the target, so we can use IFFT basis as the sparse basis $\Psi$. At last, the HRRP of the target can be reconstructed with the combination of sparse features. 
At present, there are a lot of compressed sensing algorithms based on the joint sparse model, which is based on the improvement of the greedy algorithm, and uses the joint information of all signals in the search for common support set. Here, an improved algorithm based on OMP algorithm DC-OMP (Distributed and collaborative Orthogonal Matching Pursuit) is presented, which can be used to solve such joint sparse model. The main process of the DC-OMP algorithm is proposed in [7][8].

\section{Simulation Analysis}

The effectiveness of this method is verified by experiments in this section. Assuming the transmitted signal is a random sparse stepping frequency signal. The Carrier frequency $f_{0}=10 \mathrm{GHz}$, $\Delta f=3 \mathrm{MHz}$, Pulse width $T=5 \mathrm{us}, \mathrm{PRF}=100 \mathrm{~Hz}, R_{0}=50 \mathrm{Km}, N_{a}=300, N=256, M=128$, The Relative position are [l $\left.\begin{array}{lll}0 & 2.5\end{array}\right] \mathrm{m}$, The magnitude of scattering centers are [1 0.50 .1$]$.

Experiment 1 Anti-noise performance analysis

Assumed the SNR is [051015]dB respectively, using OMP algorithm as the comparison algorithm. The HRRP results and the reconstruction error curves showed in Figure 1.

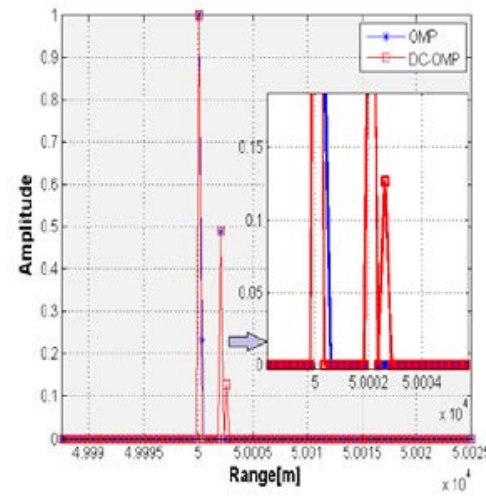

(a) SNR is OdB

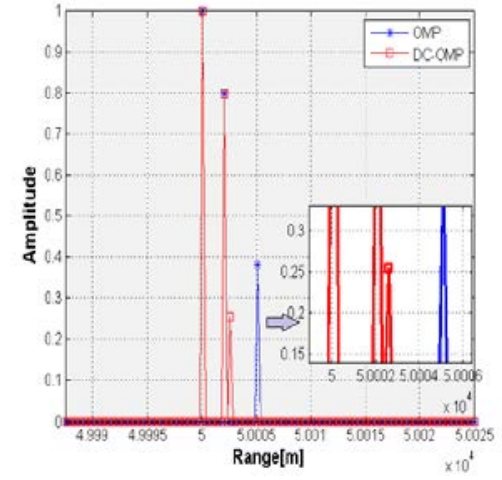

(b) SNR is $-5 \mathrm{~dB}$

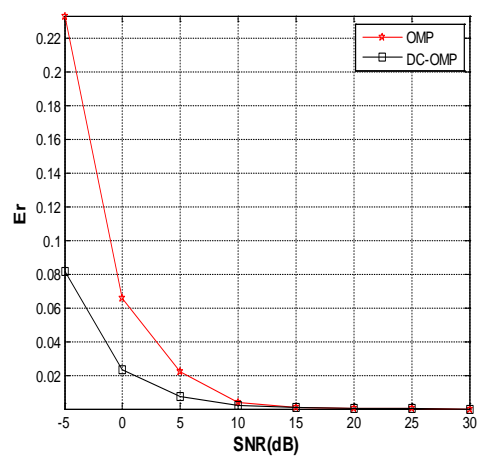

(c) Reconstruction error curves

Fig. 1 the image results and the reconstruction error curves obtained by different algorithms

The signal-to-noise ratio of $0 \mathrm{~dB},-5 \mathrm{~dB}$ for the reconstruction of the results are shown in Fig. 1(a)-(b) respectively. The reconstruction error curves under different SNR conditions are shown in Fig. 1(c). It can be seen that when the signal to noise ratio is $0 \mathrm{~dB}$, the OMP algorithm can't reconstruct with less intensity of scattered points, and the reconstruction error occurs, while the DC-OMP algorithm can reconstruct the weak scattering point accurately. When the SNR is low to $-5 \mathrm{~dB}$, DC-OMP algorithm can still accurately reconstruct the weak scattering point, showing the strong anti-noise performance.

Experiment 2 The impact of measurement value

In this section, the influence of different measurement values on the reconstruction probability is considered. If other conditions remain unchanged, the signal to noise ratio is $10 \mathrm{~dB}$, set the different measurement values, and the HRRP and the reconstructed error are shown in Figure 2.

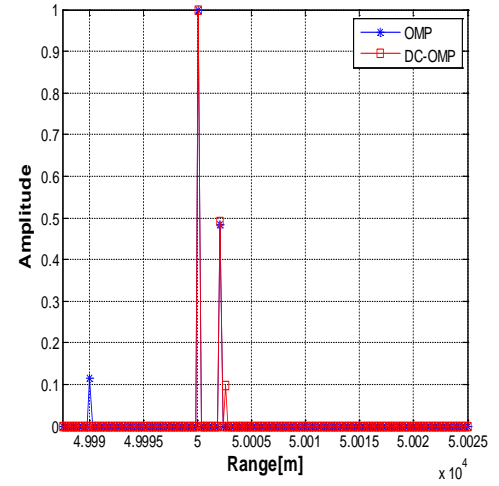

(a) $\mathrm{M}$ is 64

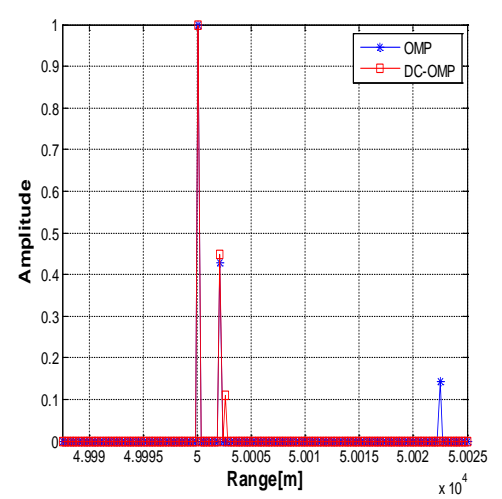

(b) $\mathrm{M}$ is 32

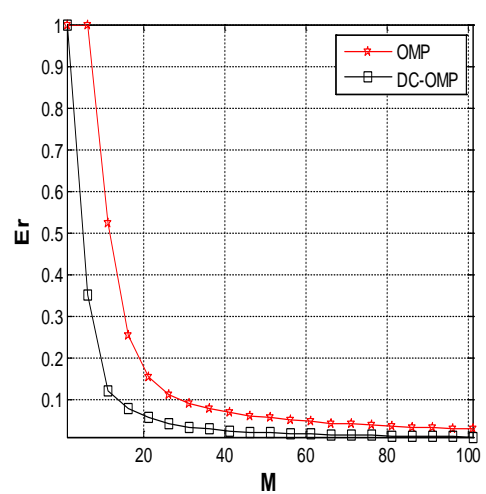

(c) The influence of measurement value

Fig. 2 The influence of measurement value on the reconstruction performance 
Fig. 2(a)-(b) were measured in different conditions to reconstruct the HRRP results. Comparison results of the reconstruction error under different measurement conditions are shown in fig. 2 (c). From Figure 2 (a) - (b) can be seen, the measurement number M dropped to 64, 32, OMP algorithm has not been properly estimated, while the DC-OMP algorithm based on signal sparse model can accurately reconstruct the HRRP result. From Fig. 2(c), it can be seen that the DC-OMP algorithm can get smaller reconstruction error under different conditions, especially in the case of small measurement value, so the method can obtain accurate reconstruction results with lower value.

\section{Summary}

In this paper, we propose a HRRP reconstruction method based on signal joint sparse model. DC-OMP algorithm is used to reconstruct the HRRP. This method has higher reconstruction accuracy, can reduce the signal measurement quantity and has stronger anti-noise performance. The simulation analysis shows the effectiveness of this method. The next step is to study the method of azimuth imaging based on the joint of sparse model, and finally get the results of $2 \mathrm{D}$ imaging and improve the imaging quality.

\section{Reference}

[1] Feng Zhu, Qun Zhang, Qiang Lei. Reconstruction of Moving Target's HRRP Using Sparse Frequency-Stepped Chirp Signal[J]. IEEE SENSORS JOURNAL, 2011, 11(10),p 2327-2334.

[2] Zhu F, Zhang Q, Luo Y, et al.. A novel cognitive ISAR imaging method with random stepped frequency chirp signal[J]. Sci China Inf Sci, 2012, 55,p1910-1924.

[3] Xu Dan-lei, Du Lan, Liu Hong-wei, et al.. Compressive Sensing Using Complex Factor Analysis for Stepped-frequency Data[J]. Journal of Electronics \& Information Technology, 2015, 37(2),p 315-321.

[4] He Jin, Luo Ying, Zhang Qun, et al.. Waveform Design and Imaging Algorithm Research of Random Frequency Stepped Chirp Signal ISAR[J]. Journal of Electronics \& Information Technology, 2011, 33(9),p2068-2075.

[5] Chen Yi-chang, Zhang Qun, Chen Xiao-pin, et al.. An Imaging Algorithm of Sparse Stepped frequency SAR Based on Multiple Measurement Vectors Model [J]. Journal of Electronics \& Information Technology, 2014, 36(12),p2986-2993.

[6] Long Teng, Mao Er-ke, He Pei-kun. Analysis and Processing of Modulated Frequency Stepped Radar Signal[J]. ACTA ELECTRONICA SINICA, 1998,12(12),p84-88.

[7] Thakshila Wimalajeewa, Pramod K. Varshney. Cooperative Sparsity Pattern Recovery in Distributed Networks Via Distributed-OMP[J]. http://arxiv.org/abs/1211.6719v1, 2012.

[8] Dennis Sundman, Greedy Algorithms for Distributed Compressed Sensing[D]. SWEDEN: KTH Royal Institute of Technology, 2014 